DIGITALCOMMONS

@WAYNESTATE-

Journal of Modern Applied Statistical Methods

\title{
The Overall F-tests for Seasonal Unit Roots under Nonstationary Alternatives: Some Theoretical Results and a Monte Carlo Investigation
}

Ghassen El Montasser

Manouba University, École Superieure de Commerce de Tunis, Tunisia, g.elmontasser@gmail.com

Follow this and additional works at: http://digitalcommons.wayne.edu/jmasm

Part of the Applied Statistics Commons, Social and Behavioral Sciences Commons, and the Statistical Theory Commons

\section{Recommended Citation}

Montasser, Ghassen El (2011) "The Overall F-tests for Seasonal Unit Roots under Nonstationary Alternatives: Some Theoretical Results and a Monte Carlo Investigation," Journal of Modern Applied Statistical Methods: Vol. 10 : Iss. 1 , Article 13.

DOI: $10.22237 /$ jmasm/1304223120

Available at: http://digitalcommons.wayne.edu/jmasm/vol10/iss1/13

This Regular Article is brought to you for free and open access by the Open Access Journals at DigitalCommons@WayneState. It has been accepted for inclusion in Journal of Modern Applied Statistical Methods by an authorized editor of DigitalCommons@WayneState. 


\title{
The Overall F-tests for Seasonal Unit Roots under Nonstationary Alternatives: Some Theoretical Results and a Monte Carlo Investigation
}

\author{
Ghassen El Montasser \\ Manouba University, \\ École Superieure de Commerce de Tunis, Tunisia
}

In many empirical studies concerning seasonal time series, it has been shown that the whole set of unit roots associated with seasonal random walks are not present. This article focuses on the overall F-tests for seasonal unit roots under some nonstationary alternatives different from the seasonal random walk. The asymptotic theory of these tests is established for these cases using a new approach based on circulant matrix concepts. The simulation results joined to this theoretic analysis showed that the overall F-tests, as well as their augmented versions, maintained high power against the nonstationary alternatives.

Key words: Kunst test, nonstationary alternatives, Brownian motion, Monte Carlo Simulation.

\section{Introduction}

The stochastic nature of seasonality appears to be gaining ground in empirical studies. Several aspects related to seasonal unit root tests are treated in the literature. In this respect, the power of these tests against nonstationary alternatives is an important issue that recently acquired some concern. To the best of our knowledge, Ghysels, Lee and Noh (1994) are the first authors who studied this question. Using a Monte Carlo study, they showed that, against a nonseasonal random walk, the power of the tests of Dickey, Hasza and Fuller (1984) is much lower than that of the tests introduced by Hylleberg, Engle, Granger and Yoo (1990).

Ghysels, et al. (1994) suggested that "the Dickey et al. test may not separate unit roots at each frequency" (p. 432). The restriction behind the Dickey, et al. procedure is that all unit roots (conventional and seasonal roots) are inseparably present with equal modulus; thus, it is clear that the conventional random walk does not fulfil this requirement. However, Rodrigues and Osborn (1999) showed that if this restriction

Ghassen El Montasser is an Assistant Professor in the Ecole Supérieure de Commerce de Tunis. Email: g.elmontasser@gmail.com. holds, the power of the Dickey, et al. tests would have a proper superiority in finite samples as opposed to that of the tests of Hylleberg, et al. (1990). Taylor (2003) analyzed the large sample behaviour of the seasonal unit root tests of Dickey, et al. when the data generating process (DGP) is a conventional random walk, that is, when the series only admits a zero frequency unit root. In such a case (and as shown by Taylor, 2003), the Dickey, et al. statistics have nondegenerate limiting distributions. These results theoretically explain the empirical findings of Ghysels, Lee and Noh (1994). Furthermore, Taylor (2005) showed that asymptotically the statistics of the Dickey, et al. augmented test will also do not diverge.

In a similar context, del Barrio Castro (2006) generalized the results of Taylor (2003) to a set of nonstationary alternatives which include the non seasonal random walk. He found that the Dickey, et al. statistics did not have standard limiting distributions and did not diverge. Based on the same methodology, del Barrio Castro (2007) established the limit theory of the Fisher and Student statistics originally developed by the Hylleberg, Engle, Granger and Yoo (1990) procedure. In that case, del Barrio Castro derived the effect that can have one unit root asymptotically on the others at different frequencies. Following the terminology of Busetti and Taylor (2003), this situation may be said to have "unattended unit roots" (p. 33). 


\section{F-TESTS FOR SEASONAL UNIT ROOTS UNDER NON-STATIONARY ALTERNATIVES}

However, del Barrio Castro (2007), in a large sample analysis, did not directly consider the effects of nonstationary alternatives on the overall F-type statistic of seasonal integration which is complementarily specified for the Hylleberg, Engle, Granger and Yoo (1990) procedure by Ghysels, Lee and Noh (1994). This article adopts the seasonal integration definition of Ghysels and Osborn (2001, p. 43).

In a recent article, Osborn and Rodrigues (2002) developed an appealing approach for deriving asymptotic results for test statistics in seasonal models with unit roots. Such an approach is based on the use of circulant matrices which could, in seasonal context, retrieve the limit theory of the involved statistics as well as conveniently traducing the dynamics of time series and its evolution across different seasons. In a similar vein, Haldrup, Montanes and Sanso (2005) have used this approach to show the effects of outliers on the limit theory of seasonal unit root tests.

This article focuses on the large sample properties of the overall F-tests of the seasonal integration when the observed series is generated from nonstationary alternatives treated by del Barrio Castro (2006). This task is accomplished using the circulant matrix-based approach of Osborn and Rodrigues (2002).

The Kunst Test

The Kunst test for quarterly time series is based on the following regression

$$
\begin{gathered}
\Delta_{4} y_{t}=\alpha_{1} y_{t-1}+\ldots+\alpha_{3} y_{t-3}+\delta y_{t-4}+\varepsilon_{t}, \\
t=1, \ldots, T
\end{gathered}
$$

which is an F-type test of the form

$$
F_{\hat{\alpha}_{1}, \ldots, \hat{\alpha}_{3}, \hat{\delta}}^{*}=(T-4)\left(\hat{\varepsilon}_{0}^{\prime} \hat{\varepsilon}_{0}-\hat{\varepsilon}^{\prime} \hat{\varepsilon}\right) /\left(\hat{\varepsilon}^{\prime} \hat{\varepsilon}\right),
$$

where $\hat{\varepsilon}_{0}$ and $\hat{\varepsilon}_{1}$ are vectors of residuals estimated under the null $H_{0}: \alpha_{1}=\ldots=\alpha_{3}=\delta=0$ and alternative hypotheses of the test. Assuming that, without any loss of generality, the initial values required by (1) are null. It should be noted that Kunst did not divide the numerator of the statistic (2) by 4 (the number of restrictions), as was done in this research to perform a conventional Fisher test.

The Hylleberg, Engle, Granger and Yoo (HEGY) Test

The basic regression for the HEGY test, without any augmentation and with no deterministic terms, is:

$$
\begin{gathered}
\Delta_{4} y_{t}=\pi_{1} y_{1 t-1}+\pi_{2} y_{2 t-1}+\pi_{3} y_{3 t-2}+\pi_{4} y_{3 t-1}+\varepsilon_{t}, \\
t=1, \ldots, T
\end{gathered}
$$

where

$$
\begin{gathered}
y_{1 t}=\left(1+L+L^{2}+L^{3}\right) y_{t}, \\
y_{2 t}=-\left(1-L+L^{2}-L^{3}\right) y_{t}, \\
y_{3 t}=-\left(1-L^{2}\right) y_{t},
\end{gathered}
$$

with $L$ as the lag operator.

Ghysels, Lee and Noh (1994) extended the HEGY approach with a joint test statistic $F_{1234}$ for the null hypothesis, $H_{0}: \pi_{1}=\pi_{2}=\pi_{3}=\pi_{4}=0$, implying all unit roots in data are observed at quarterly frequency. $H_{0}$ is an overall hypothesis for seasonal integration SI (1) in accordance with the notation of Ghysels and Osborn (2001). Note that:

$$
\left[\begin{array}{l}
y_{1 t-1} \\
y_{2 t-1} \\
y_{3 t-2} \\
y_{3 t-1}
\end{array}\right]=\left[\begin{array}{cccc}
1 & 1 & 1 & 1 \\
-1 & 1 & -1 & 1 \\
0 & -1 & 0 & 1 \\
-1 & 0 & 1 & 0
\end{array}\right]\left[\begin{array}{l}
y_{t-1} \\
y_{t-2} \\
y_{t-3} \\
y_{t-4}
\end{array}\right] .
$$

It may be deduced from (5) that the regressors of the Kunst test are non-singular linear transformations of those of the HEGY test. Consequently, the F-type statistics, $F_{1234}$ and $F_{\hat{\alpha}_{1}, \ldots, \hat{\alpha}_{3}, \hat{\delta}}^{*} / 4$, will have the same limit theory. Given that the two statistics are asymptotically related, the analysis is confined to that of Kunst in the sequel. 


\section{EL MONTASSER}

It can be observed that slight differences exist between the critical values of both statistics. In general, such critical values are tabulated assuming that the DGP of $y_{t}$ is:

$$
y_{t}=y_{t-4}+e_{t} \text {. }
$$

In this article it is assumed that the DGP of $y_{t}$ is drawn from one of the following stochastic processes:

$$
\begin{aligned}
& y_{t}=y_{t-1}+e_{t}, \\
& y_{t}=-y_{t-1}+e_{t}, \\
& y_{t}=y_{t-2}+e_{t}, \\
& y_{t}=-y_{t-2}+e_{t},
\end{aligned}
$$

or

$$
y_{t}=-y_{t-1}-y_{t-2}-y_{t-3}+e_{t} \text {. }
$$

Using the double subscript notation, the following annual vectors can be defined:

$$
Y_{n}=\left(y_{1 n}, y_{2 n}, y_{3 n}, y_{4 n}\right)^{\prime},
$$

and

$$
E_{n}=\left(e_{1 n}, e_{2 n}, e_{3 n}, e_{4 n}\right)^{\prime},
$$

where it is assumed that $n=1, \ldots, N$ and in $T$ observations there are $N$ years, thus, $T=4 N$. To keep matters tractable, suppose that $Y_{0}=\left(y_{10}, y_{20}, y_{30}, y_{40}\right)^{\prime}=(0,0,0,0)^{\prime}$.

The error processes in the alternatives (A.1)-(A.5) follow a stationary $\mathrm{AR}(\mathrm{p})$

$$
\phi(L) e_{s n}=v_{s n},
$$

where $\varphi(z) e_{s n}=1-\sum_{i=1}^{p} \phi_{i} z^{i}$ and $\mathrm{s}=1, \ldots, 4$.

The roots of $\varphi(z)=0$ all lie outside the unit circle $|z|=1$. As for the error sequence $\left\{v_{s n}\right\}$, it depicts an innovation process with constant conditional variance $\sigma^{2}$ (see Spanos,
2003, p. 443). Similar to what has been conjectured by del Barrio Castro (2007) regarding the error structure in the nonstationary alternatives described above, suppose that the vector $E_{n}$ has the following dynamics:

$$
E_{n}=\sum_{j=0}^{\infty} \Gamma_{j}^{*} V_{n}
$$

where $v_{n}=\left(v_{1 n}, v_{2 n}, v_{3 n}, v_{4 n}\right)^{\prime}, \quad$ and the sequence of $4 \times 4$ matrices are defined as:

$$
\begin{gathered}
\Gamma_{0}^{*}=\left[\begin{array}{cccc}
1 & 0 & 0 & 0 \\
\gamma_{1} & 1 & 0 & 0 \\
\gamma_{2} & \gamma_{1} & 1 & 0 \\
\gamma_{3} & \gamma_{2} & \gamma_{1} & 1
\end{array}\right] \\
\Gamma_{0}^{*}=\left[\begin{array}{cccc}
\gamma_{j 4} & \gamma_{j 4-1} & \gamma_{j 4-2} & \gamma_{j 4-3} \\
\gamma_{j 4+1} & \gamma_{j 4} & \gamma_{j 4-1} & \gamma_{j 4-2} \\
\gamma_{j 4+2} & \gamma_{j 4+1} & \gamma_{j 4} & \gamma_{j 4-1} \\
\gamma_{j 4+3} & \gamma_{j 4+2} & \gamma_{j 4+1} & \gamma_{j 4}
\end{array}\right],
\end{gathered}
$$

for $j=1,2, \ldots .$. with

$$
\gamma(z)=1-\sum_{j=1}^{\infty} \gamma_{j} z^{j}
$$

being the inverse of $\varphi(z)$. Finally, $\Gamma^{*}(1)$ is defined as:

$$
\Gamma^{*}(1)=\sum_{j=0}^{\infty} \Gamma_{j}^{*} .
$$

del Barrio Castro (2006) used the vector of moving average representation to express the alternatives (A.i), i $=1, \ldots, 5$, in a vector of quarters representations where the observations of each year are stacked in the above defined vectors $Y_{n}$ et $E_{n}$, let

$$
(1-B) Y_{n}=\left(\Theta_{0}^{i}+\Theta_{1}^{i} B\right) E_{n}, \quad i=1,2, \ldots, 5,
$$




\section{F-TESTS FOR SEASONAL UNIT ROOTS UNDER NON-STATIONARY ALTERNATIVES}

where $\mathrm{B}$ is the annual backward operator. The $4 \times 4$ matrices $\Theta_{0}^{i}$ and $\Theta_{1}^{i}$ (corresponding to the alternatives A.1-A.5) are defined as follows: for (A.1)

$$
\Theta_{0}^{1}=\left[\begin{array}{llll}
1 & 0 & 0 & 0 \\
1 & 1 & 0 & 0 \\
1 & 1 & 1 & 0 \\
1 & 1 & 1 & 1
\end{array}\right], \Theta_{1}^{1}=\left[\begin{array}{llll}
0 & 1 & 1 & 1 \\
0 & 0 & 1 & 1 \\
0 & 0 & 0 & 1 \\
0 & 0 & 0 & 0
\end{array}\right],
$$

for (A.2)

$$
\begin{aligned}
& \Theta_{0}^{2}=\left[\begin{array}{cccc}
1 & 0 & 0 & 0 \\
-1 & 1 & 0 & 0 \\
1 & -1 & 1 & 0 \\
-1 & 1 & -1 & 1
\end{array}\right], \\
& \Theta_{1}^{1}=\left[\begin{array}{cccc}
0 & -1 & 1 & -1 \\
0 & 0 & -1 & 1 \\
0 & 0 & 0 & -1 \\
0 & 0 & 0 & 0
\end{array}\right],
\end{aligned}
$$

for (A.3)

$$
\Theta_{0}^{3}=\left[\begin{array}{llll}
1 & 0 & 0 & 0 \\
0 & 1 & 0 & 0 \\
1 & 0 & 1 & 0 \\
0 & 1 & 0 & 1
\end{array}\right], \Theta_{1}^{3}=\left[\begin{array}{llll}
0 & 0 & 1 & 0 \\
0 & 0 & 0 & 1 \\
0 & 0 & 0 & 0 \\
0 & 0 & 0 & 0
\end{array}\right],
$$

for (A.4)

$$
\begin{aligned}
& \Theta_{0}^{4}=\left[\begin{array}{cccc}
1 & 0 & 0 & 0 \\
0 & 1 & 0 & 0 \\
-1 & 0 & 1 & 0 \\
0 & -1 & 0 & 1
\end{array}\right], \\
& \Theta_{1}^{4}=\left[\begin{array}{cccc}
0 & 0 & -1 & 0 \\
0 & 0 & 0 & -1 \\
0 & 0 & 0 & 0 \\
0 & 0 & 0 & 0
\end{array}\right],
\end{aligned}
$$

and for (A.5)

$$
\begin{gathered}
\Theta_{0}^{5}=\left[\begin{array}{cccc}
1 & 0 & 0 & 0 \\
-1 & 1 & 0 & 0 \\
0 & -1 & 1 & 0 \\
0 & 0 & -1 & 1
\end{array}\right], \\
\Theta_{1}^{5}=\left[\begin{array}{llll}
0 & 0 & 0 & -1 \\
0 & 0 & 0 & 0 \\
0 & 0 & 0 & 0 \\
0 & 0 & 0 & 0
\end{array}\right] .
\end{gathered}
$$

The following result was established by del Barrio Castro (2007)

$$
\begin{gathered}
\frac{1}{\sigma \sqrt{N}} Y_{[r N]} \rightarrow_{d} B_{i}(r), \\
B_{i}(r)=C_{i} \Gamma^{*}(1) B(r), \\
C_{i}=\Theta_{0}^{i}+\Theta_{1}^{i} \\
i=1,2, \ldots, 5
\end{gathered}
$$

where the symbol $\rightarrow_{d}$ denotes the convergence of probability measures, $B_{i}(r)$ is a $4 \times 1$ vector Brownian motion process with variance matrix $\Omega_{i}=\sigma^{2} C_{i} \Gamma^{*}(1) \Gamma^{*}(1)^{\prime} C_{i}^{\prime}$ and $B(r)$ is a vector Brownian motion with variance matrix $\sigma^{2} I_{4}$. The subscript $i$ corresponds to the alternative (A.i), $i=1, \ldots, 5$.

Note that the rank of $C_{i}, i=1, \ldots, 5$, is the number of (seasonal) unit roots implied by the process (A.i), $i=1, \ldots, 5$. In order to determine the number of cointegration relations between the quarters corresponding to every process (A.i), $\mathrm{i}=1, \ldots, 5$, it is necessary to subtract from the periodicity of the quarterly data, that is 4 , the rank of the matrix $C_{i}$, $i=1, \ldots, 5$. Equation (8) may be rewritten more precisely by identifying the stochastic processes $B_{i}(r), i=1,2, . .5$, on the grounds that there is always cointegration among the quarters of the time series (see del Barrio Castro, 2007, p.915). Limit Theory of the Kunst Test Under Nonstationary Alternatives 


\section{EL MONTASSER}

The following lemma can be directly deduced from the preceding result of del Castro Barrio (2007) and lemma A.1 of Osborn and Rodrigues (2002).

Lemma

Supposing that the DGP of $y_{t}$ in (1) is given by the alternatives (A.1)-(A.5) and also that the vector $\left(e_{1 n}, \ldots, e_{4 n}\right), \forall n$, satisfies assumption 1 of Phillips (1986, p.313), then under the null of the Kunst test is $T \rightarrow \infty$

(a)

$$
\begin{gathered}
N^{-2} \sum_{n=1}^{N} Y_{n} Y_{n}^{\prime} \rightarrow_{d} \sigma^{2} \int_{0}^{1} M_{i} B(r) B(r)^{\prime} M_{i}^{\prime} d r \\
i=1,2, \ldots, 5
\end{gathered}
$$

(b)

$$
\begin{gathered}
N^{-1} \sum_{n=1}^{N} Y_{n-1} \varepsilon_{n}^{\prime} \rightarrow_{d} \sigma^{2} M_{i} \int_{0}^{1} B(r) d B(r)^{\prime} M_{i}^{\prime}, \\
i=1,2, \ldots, 5 .
\end{gathered}
$$

(c)

$$
\begin{gathered}
T^{-2} \sum_{t=1}^{T} y_{t-k}^{2} \rightarrow_{d} \frac{\sigma^{2}}{16} \int_{0}^{1} B(r)^{\prime} M_{i}^{\prime} M_{i} B(r) d r \\
k=1, \ldots, 4, \quad i=1,2, \ldots, 5
\end{gathered}
$$

(d)

$T^{-2} \sum_{t=1}^{T} y_{t-k} y_{t-j} \rightarrow_{d} \frac{\sigma^{2}}{16} \int_{0}^{1} B(r)^{\prime} M_{i}^{\prime} H_{k}^{\prime} H_{j} M_{i} B(r) d r$,

$$
k \neq j, i=1,2, \ldots, 5 \text {. }
$$

(e)

$$
\begin{gathered}
T^{-1} \sum_{t=1}^{T} y_{t-k} \varepsilon_{t} \rightarrow_{d} \frac{\sigma^{2}}{4} \int_{0}^{1} B(r)^{\prime} M_{i}^{\prime} H_{k}^{\prime} M_{i} d B(r), \\
k=1, \ldots, 4, \quad i=1,2, \ldots, 5
\end{gathered}
$$

where

$$
\varepsilon_{n}=\left(\varepsilon_{1 n}, \varepsilon_{2 n}, \varepsilon_{3 n}, \varepsilon_{4 n}\right)^{\prime}
$$

and

$$
M_{i}=C_{i} \Gamma^{*}
$$

The matrix $H_{k}, k=1,2,3,4$, is a particular permutation matrix order 4 which produces the following elementary operations: let a matrix $K$ have 4 lines, the operation $H_{1} K$ moves the last row of $K$ to the top row of $H_{1} K$ and the other rows move down one place. More generally, $H_{i} K$ shifts the final $i^{\text {th }}$ rows to the top of the matrix while the remaining rows correspondingly move down; note that $H_{4}=I_{4}$ (see Golub \& Van Loan, 1996, p. 109112 , for details). The OLS estimator $\hat{\alpha}$ of the vector $\alpha=\left(\alpha_{1}, \alpha_{2}, \alpha_{3}, \delta\right)^{\prime}$ defined in Equation (1) satisfies under the null of Kunst test the asymptotic results represented by the following theorem.

Theorem

If that the DGP of $y_{t}$ in (1) is given by one of the alternatives (A.1) - (A.5), then:

(a)

$$
\frac{T}{4}(\hat{\alpha}-\alpha) \rightarrow_{d} F^{-1} f
$$

(matrices for $F$ and $f$ are shown in Figure 1);

(b) The Student statistic $t_{\hat{\alpha}_{i}}$ corresponding to the $\mathrm{i}^{\text {th }}$ component of vector $\hat{\alpha}$ satisfies the following result:

$$
t_{\hat{\alpha}_{i}} \rightarrow_{d} \frac{\left(F^{-1} f\right)_{i}}{\left(\left(F^{-1}\right)_{i i}\right)^{1 / 2}}
$$

and

(c) The F-type statistic of Kunst test verifies:

$$
F_{\hat{\alpha}_{1}, \ldots, \hat{\alpha}_{3}, \hat{\delta}}^{*} \rightarrow_{d} f^{\prime} F^{-1} f .
$$




$$
\begin{aligned}
& \text { Figure 1: } F \text { and } f \text { Matrices for } \frac{T}{4}(\hat{\alpha}-\alpha) \rightarrow_{d} F^{-1} f \\
& F=\left[\begin{array}{cccc}
\int_{0}^{1} B(r)^{\prime} M_{i}^{\prime} H_{1}^{\prime} H_{1} M_{i} B(r) d r & \int_{0}^{1} B(r)^{\prime} M_{i}^{\prime} H_{1}^{\prime} H_{2} M_{i} B(r) d r & \int_{0}^{1} B(r)^{\prime} M_{i}^{\prime} H_{1}^{\prime} H_{3} M_{i} B(r) d r & \int_{0}^{1} B(r)^{\prime} M_{i}^{\prime} H_{1}^{\prime} M_{i} B(r) d r \\
\int_{0}^{1} B(r)^{\prime} M_{i}^{\prime} H_{2}^{\prime} H_{1} M_{i} B(r) d r & \int_{0}^{1} B(r)^{\prime} M_{i}^{\prime} H_{2}^{\prime} H_{2} M_{i} B(r) d r & \int_{0}^{1} B(r)^{\prime} M_{i}^{\prime} H_{2}^{\prime} H_{3} M_{i} B(r) d r & \int_{0}^{1} B(r)^{\prime} M_{i}^{\prime} H_{2}^{\prime} M_{i} B(r) d r \\
\ldots & \ldots & \ldots & \ldots \\
\int_{0}^{1} B(r)^{\prime} M_{i}^{\prime} H_{1} M_{i} B(r) d r & \int_{0}^{1} B(r)^{\prime} M_{i}^{\prime} H_{2} M_{i} B(r) d r & \int_{0}^{1} B(r)^{\prime} M_{i}^{\prime} H_{3} M_{i} B(r) d r & \int_{0}^{1} B(r)^{\prime} M_{i}^{\prime} M_{i} B(r) d r
\end{array}\right] \\
& f=\left[\begin{array}{c}
\int_{0}^{1} B(r)^{\prime} M_{i}^{\prime} H_{1}^{\prime} M_{i} d B(r) \\
\int_{0}^{1} B(r)^{\prime} M_{i}^{\prime} H_{2}^{\prime} M_{i} d B(r) \\
\ldots \\
\int_{0}^{1} B(r)^{\prime} M_{i}^{\prime} H_{3}^{\prime} M_{i} d B(r) \\
\int_{0}^{1} B(r)^{\prime} M_{i}^{\prime} M_{i} d B(r)
\end{array}\right] \forall i=1,2, \ldots, 5
\end{aligned}
$$

Proof

Before showing the proof of result (a) of the theorem, consider the properties of the matrix F: the elements of the main diagonal of $\mathrm{F}$ are all equal and the elements of $F$ along each diagonal line parallel to the principal diagonal are equal, thus, $\mathrm{F}$ is a Toeplitz matrix. Toeplitz matrices belong to the larger class of persymmetric matrices. A square matrix B of order $\mathrm{n}$ is persymmetric if it is symmetric about the northeast-southwest diagonal, that is, $b_{i j}=b_{n-j+1, n-i+1}$ for all $i$ and $j$. Moreover, from the properties of the matrices $H_{k}$, $k=1,2,3,4$, it can be shown that the matrix $\mathrm{F}$ is also symmetric. Equation (1) can be written in matrix form:

$$
Y=X \alpha+\varepsilon
$$

where

$$
X=\left[\begin{array}{cccc}
y_{0} & y_{-1} & y_{-2} & y_{-3} \\
y_{1} & y_{0} & y_{-1} & y_{-2} \\
\cdots & \cdots & \cdots & \cdots \\
y_{T-1} & y_{T-2} & y_{T-3} & y_{T-4}
\end{array}\right]
$$

and

$$
\mathcal{E}=\left[\begin{array}{c}
\mathcal{E}_{1} \\
\cdots \\
\mathcal{E}_{T}
\end{array}\right] .
$$

In addition, $\quad \hat{\alpha}-\alpha=\left(X^{\prime} X\right)^{-1} X^{\prime} \varepsilon$,

where

$X^{\prime} X=$

$\left[\begin{array}{cccc}\sum_{1}^{T} y_{t-1}^{2} & \sum_{1}^{T} y_{t-1} y_{t-2} & \sum_{1}^{T} y_{t-1} y_{t-3} & \sum_{1}^{T} y_{t-1} y_{t-4} \\ \sum_{1}^{T} y_{t-2} y_{t-1} & \sum_{1}^{T} y_{t-2}^{2} & \sum_{1}^{T} y_{t-2} y_{t-3} & \sum_{1}^{T} y_{t-2} y_{t-4} \\ \sum_{1}^{T} y_{t-3} y_{t-1} & \sum_{1}^{T} y_{t-3} y_{t-2} & \sum_{1}^{T} y_{t-3}^{2} & \sum_{1}^{T} y_{t-3} y_{t-4} \\ \sum_{1}^{T} y_{t-4} y_{t-1} & \sum_{1}^{T} y_{t-4} y_{t-2} & \sum_{1}^{T} y_{t-4} y_{t-3} & \sum_{1}^{T} y_{t-4}^{2}\end{array}\right]$,

and

$$
X^{\prime} \mathcal{\varepsilon}=\left[\begin{array}{c}
\sum_{1}^{T} y_{t-1} \varepsilon_{t} \\
\cdots \\
\sum_{1}^{T} y_{t-4} \varepsilon_{t}
\end{array}\right] .
$$




\section{EL MONTASSER}

Also: $\frac{T}{4}(\hat{\alpha}-\alpha)=\frac{1}{4}\left(\frac{X^{\prime} X}{T^{2}}\right)^{-1} \frac{X^{\prime} \varepsilon}{T}$ and, due to parts (c), (d) and (e) of the preceding lemma and the fact that $H_{4}=I_{4}$, the result of the theorem holds.

The asymptotic distributions of Student statistics corresponding to the parameters of Equation 1 can be deduced from result (a) of the theorem. To prove result (c), the F-type statistic of Kunst can be written as follows: $F_{\hat{\alpha}_{1}, \ldots, \hat{\alpha}_{3}, \hat{\delta}}^{*}=\hat{\alpha}^{\prime}\left[\left(S^{2}\right)^{-1} X^{\prime} X\right] \hat{\alpha}$, where $S^{2}$ is the OLS estimator of the residual variance in Equation (1). The (Toeplitz) circulant matrix F and its inverse are symmetric, consequently result (c) holds and the theorem is proved.

Empirical quantiles of the Kunst test for the processes (A.1)-(A.5) were generated and associated with nominal levels $90 \%, 95 \%$ and $99 \%$. The sample size considered is $4,000(1,000$ years) with 20,000 replications; it has been shown that these empirical quantiles tend to be infinite (these results are not presented, but are available upon request). Consequently, it is possible to predict that in $100 \%$ of cases the null hypothesis will be rejected for the processes (A.1)-(A.5) for nominal levels of $5 \%$ and $1 \%$. Table 1 shows the rejection frequencies for a sample size of 100 (25 years) and 20,000 replications. All simulations were conducted using the software Matlab.
In addition, augmented regression (1) corresponding to the Kunst test was carried out by lagged values of the independent variable, thus, this regression becomes

$$
\begin{gathered}
\Delta_{4} y_{t}=\alpha_{1} y_{t-1}+\ldots+\alpha_{3} y_{t-3}+\delta y_{t-4}+\sum_{i=1}^{p} \Delta_{4} y_{t-i}+\varepsilon_{t} \\
t=1, \ldots, T .
\end{gathered}
$$

Table 2 reports the power of the augmented Kunst test against the nonstationary alternatives (A.1) - (A.5). Results in Table 2 show that perfect power is maintained across all the alternatives (A.1) - (A.5) even if the number of lagged terms of the dependent variable increases. At this level, a slight exception to this general finding was detected for the alternative (A.5) and for $\mathrm{p}=4$ or $\mathrm{p}=6$. Particularly, and for this alternative, the exception is much clearer for $p=6$ and the nominal level $1 \%$. In fact, the test power decreases and reaches a value of approximately $66 \%$.

\section{Conclusion}

A large amount of literature on testing for seasonal unit roots has appeared during the last two decades. However, the majority of econometricians treating this topic have bent over backwards to give the limit theory of the tests for unit roots at the zero, Nyquist and

Table 1: Empirical Rejection Frequencies of Kunst Test under Nonstationary Alternatives

\begin{tabular}{|c|c|c|c|c|c|c|}
\hline & \multicolumn{5}{|c|}{ Processes } \\
\cline { 2 - 7 } Kunst Test & (A.0) & (A.1) & (A.2) & (A.3) & (A.4) & (A.5) \\
\hline $\begin{array}{c}F_{\hat{\alpha}_{1}, \ldots, \hat{\alpha}_{3}, \hat{\delta}}^{*} \\
\text { Nominal Size } \\
5 \%\end{array}$ & 0.095 & 1 & 1 & 1 & 1 & 1 \\
\hline $\begin{array}{c}F_{\hat{\alpha}_{1}, \ldots, \hat{\alpha}_{3}, \hat{\delta}}^{*} \\
\text { Nominal Size } \\
1 \%\end{array}$ & 0.0158 & 1 & 1 & 1 & 1 & 1 \\
\hline
\end{tabular}

Number of replications: 20,000; Sample size 4N = 100 observations 


\section{F-TESTS FOR SEASONAL UNIT ROOTS UNDER NON-STATIONARY ALTERNATIVES}

Table 2: Empirical Rejection Frequencies of the Kunst Augmented Test under Nonstationary Alternatives

\begin{tabular}{|l|c|c|c|c|c|c|c|}
\cline { 3 - 8 } \multicolumn{2}{c|}{} & \multicolumn{7}{c|}{ Processes } \\
\cline { 2 - 8 } & $(\mathrm{A} .0)$ & $(\mathrm{A} .1)$ & $(\mathrm{A} .2)$ & $(\mathrm{A} .3)$ & $(\mathrm{A} .4)$ & $(\mathrm{A} .5)$ \\
\hline \multirow{3}{*}{$\begin{array}{l}\text { Nominal } \\
\text { Size } 5 \%\end{array}$} & $\mathrm{p}=2$ & 0.0592 & 1 & 1 & 1 & 1 & 1 \\
\cline { 2 - 8 } & $\mathrm{p}=4$ & 0.0549 & 1 & 1 & 1 & 1 & 0.9920 \\
\hline \multirow{3}{*}{$\begin{array}{l}\text { Nominal } \\
\text { Size } 1 \%\end{array}$} & $\mathrm{p}=6$ & 0.0522 & 1 & 1 & 0.9980 & 0.9976 & 0.9038 \\
\cline { 2 - 8 } & $\mathrm{p}=4$ & 0.0141 & 1 & 1 & 0.9992 & 0.9991 & 0.9271 \\
\cline { 2 - 8 } & $\mathrm{p}=6$ & 0.0121 & 0.9990 & 0.9990 & 0.97770 & 0.97460 & 0.6632 \\
\hline
\end{tabular}

harmonic seasonal frequencies by considering either an additional determinist component or a modified hypothesis set concerning the error terms which appear in the regression models associated with such tests. Seldom have works concerning this topic studied the power of seasonal unit roots against nonstationary alternatives. Ghysels, et al. (1994) studied this problem and, in a simulation study, they hypothesized that the DHF test may not separate unit roots at each frequency. Having enriched this analysis by a large sample investigation, Taylor (2003) found that the DHF statistics did not diverge to minus infinity when the DGP of the series is a conventional random walk. del Barrio Castro (2006, 2007) considered an extended set of nonstationary alternatives and studied their asymptotic effects on the DHF and HEGY statistics.

This article extended the problem treated by Taylor (2003) and del Castro Barrio (2007) to the overall F-type tests for seasonal integration. It has been realized that the most renowned tests, that is, those of HEGY (1990) and Kunst (1997), are asymptotically related. For this reason, this research focused on asymptotic effects of the nonstationary alternatives, (A.1) - (A.5) on Kunst F-type test. To reach this goal, I had the circulant-matrixbased approach introduced by Osborn and Rodrigues (2002) was chosen. Moreover, in a simulation study, it was found that the Kunst F- type statistic maintained high power when the totality of unit roots implied by the filter $\left(1-L^{4}\right) \quad$ were not present. In addition, these high-power properties are preserved when the regression model of the test was augmented with lagged dependent variables. The approach adopted in this article can be applied to cases other than the quarterly one. To this aim, it is enough to write the adequate matrices $C_{i}$ defined from Equation (5) following the data observation frequency.

\section{References}

Busetti, F., \& Taylor, A. M. R. (2003). Testing against stochastic trend and seasonality in the presence of unattended breaks and unit roots. Journal of Econometrics, 117, 21-53.

Dickey, D. A., Hasza, D. P., \& Fuller, W. A. (1984). Testing for unit roots in seasonal time series. Journal of American Statistical Association, 79, 355-367.

del Barrio Castro, T. (2007). Using the HEGY procedure when not all roots are present. Journal of Time Series Analysis, 28, 910-922.

del Barrio Castro, T. (2006). On the performance of the DHF test against nonstationary alternatives. Statistics and Probability Letters, 76, 291-296. 


\section{EL MONTASSER}

Ghysels, E., Lee, H. S., \& Noh, J. (1994). Testing for unit roots in seasonal time series: Some theoretical extensions and a Monte Carlo investigation. Journal of Econometrics, $62,415-442$.

Ghysels, E., \& Osborn, D. R. (2001). The econometric analysis of seasonal time series. Cambridge, MA: Cambridge University Press.

Golub, G. H., \& van Loan, C. F. (1996). Matrix computations. Baltimore: The Johns Hopkins University Press.

Haldrup, N., Montanés, A., \& Sanso, A. (2005). Measurement errors and outliers in seasonal unit root testing. Journal of Econometrics, 127, 103-128.

Hylleberg, S., Engle, R. F., Granger, C. W. J., \& Yoo, B. S. (1990). Seasonal integration and cointegration. Journal of Econometrics, 44, 215-238.

Kunst, R. M. (1997). Testing for cyclical non-stationarity in autoregressive models. Journal of Time Series Analysis, 18, 123-135.
Osborn, D. R., \& Rodrigues, P. M. M. (2002). The asymptotic distributions of seasonal unit root tests: a unifying approach. Econometric Reviews, 21, 221-241.

Phillips, P. C. B. (1986). Understanding Spurious Regressions in Econometrics. Journal of Econometrcis, 33, 311-340.

Rodrigues, P. M. M., \& Osborn, D. R. (1999). Performance of seasonal unit root tests for monthly data. Journal of Applied Statistics, 26, 985-1004.

Spanos, A. (2003). Probability theory and statistical inference: econometric modeling with observational data. Cambridge MA: Cambridge University Press (Virtual Publishing).

Taylor, A. M. R. (2005). On the limiting behaviour of augmented seasonal unit root tests. Economics Bulletin, 3, 1-10.

Taylor, A. M. R. (2003). On the asymptotic properties of some seasonal unit root tests. Econometric Theory, 19, 311-321. 